Marquette University

e-Publications@Marquette

Mathematics, Statistics and Computer Science

Mathematics, Statistics and Computer Science,

Faculty Research and Publications

Department of

$10-1-2014$

\title{
Relatively Congruence-free Regular Semigroups
}

Peter Jones

Marquette University, peter.jones@marquette.edu

Accepted version. Semigroup Forum, Vol. 89, No. 2 (October 2014): 383-393. (C) 2014 Springer. Used with permission.

The final publication is available at Springer via DOI.

Shareable Link. Provided by the Springer Nature SharedIt content-sharing initiative. 


\title{
Relatively congruence-free regular semigroups
}

\author{
Peter R. Jones \\ Department of Mathematics, Statistics and Computer Science, \\ Marquette University, Marquette University \\ Milwaukee, WI
}

\section{Abstract:}

Yu, Wang, Wu and Ye call a semigroup $S T$-congruence-free, where $T$ is an equivalence relation on $S$, if any congruence $\rho$ on $S$ is either disjoint from $T$ or contains $T$. A congruence-free semigroup is then just an $\omega$-congruence-free semigroup, where $\omega$ is the universal relation. They determined the completely regular semigroups that are $T$-congruence-free with respect to each of the Green's relations.

The goal of this paper is to extend their results to all regular semigroups. Such a semigroup is $\mathcal{J}$-congruence-free if and only if it is either a semilattice or has a single nontrivial $\mathcal{J}$-class, $J$, say, and either $J$ is a subsemigroup, in which case it is congruence-free, or otherwise its principal factor is congruence-free. Given the current knowledge of congruence-free regular semigroups, this result is probably best possible. When specialized to completely semisimple semigroups, however, a complete answer is obtained, one that specializes to that of $Y u$ et al. A similar outcome is obtained for $L$ and $\mathrm{R}$. In the case of $\mathrm{H}$, only the completely semisimple case is fully resolved, again specializing to those of $\mathrm{Yu}$ et al.

Keywords: Congruence-free, Relatively congruence-free, $\mathrm{H}-\mathrm{CF}$, Completely semisimple. 
NOT THE PUBLISHED VERSION; this is the author's final, peer-reviewed manuscript. The published version may be accessed by following the link in the citation at the bottom of the page.

\section{Introduction}

Let $S$ be a semigroup and $T$ any equivalence relation on $S$. Yu et al. [1] defined $S$ to be $T$-congruence-free if any congruence $\rho$ on $S$ is either disjoint from $T$ or contains $T$; following their terminology, we abbreviate the term $T$-congruence-free to $T$-CF. A congruence-free semigroup is then just an $\omega$-CF semigroup, where $\omega$ is the universal relation, so this concept generalizes that of congruence-freedom. Although the finite congruence-free semigroups were determined long ago (see below), not much is known about congruence-free semigroups in general. It is known [5] that any semigroup can be embedded in such a semigroup; likewise (also [5]) any inverse semigroup can be embedded in a congruence-free inverse semigroup. Thus we take such semigroups as the base point of our investigation.

Here we determine the $\mathcal{J}$-CF regular semigroups, modulo congruence-free regular semigroups, and prove an analogous result for L-CF regular semigroups. (Since the L-CF and R-CF properties are dual, we make no specific mention of the latter in the sequel).

While congruence-free semigroups in general appear to be intractable, those that are completely 0-simple are well determined. Thus probably the broadest sub-class in which explicit answers may be expected is that of completely semisimple semigroups: those whose principal factors are completely [0-] simple semigroups. In Section 4 we completely determine the $\mathcal{J}-\mathrm{CF}$, L-CF and H-CF completely semisimple semigroups. These results then easily specialize to those of [1] for completely regular semigroups. Apart from the generalization Lemma 2.1 of their first key result, our methods are independent of theirs. These methods do not seem to yield useful information for D-CF (except in the completely semisimple situation, where $D=J$ ).

We continue this section with some background and preliminary results. Denote the universal and identical relations by $\omega$ and $\epsilon$ respectively. If $A$ is a subset of a semigroup $S, E_{A}$ denotes the set of idempotents in $A$. If $S$ is a regular semigroup, $V(a)$ denotes the set of inverses of the element $a$. Only elementary properties of regular semigroups, such as may be found in [3], are needed in the sequel.

Semigroup Forum, Vol. 89, No. 2 (October 2014): pg. 383-393. DOI. This article is (C) Springer and permission has been granted for this version to appear in e-Publications@Marquette. Springer does not grant permission for this article to be further copied/distributed or hosted elsewhere without the express permission from Springer. 
Let $S$ be any semigroup, for the moment. The symbols $L, R, H, D$ and J refer, as usual to Green's relations. A semigroup is combinatorial if $\mathrm{H}=\epsilon$. We extend that terminology to subsets of semigroups.

Let $J$ be a $J$-class of $S$. Denote by $I(J)$ the ideal generated by $J$, that is, $S^{1} J S^{1}$. The $\mathcal{J}$-classes of $S$ are partially ordered by $J_{1} \leq J_{2}$ if $I\left(J_{1}\right) \subseteq I\left(J_{2}\right)$. Denote by $Q(J)$ the ideal $I(J) \backslash J$, consisting of the (possibly empty) union of the $J$-classes strictly below $J$. The principal factor $P F(J)[2, \S 2.6]$ is the Rees quotient $I(J) / Q(J)$ where, by convention, if $Q(J)$ is empty (that is, $J$ is the least $J$-class of $S$ ) $P F(J)=J$. In practice, in the general case one may identify $\operatorname{PF}(J)$ with the set $J \cup\{0\}$, where if $a, b \in J$, the product is that in $S$, should it remain in $J$, and otherwise 0 .

In general, each principal factor is either a null semigroup, a 0simple semigroup or, in the case of a least $\mathcal{J}$-class, a simple semigroup. The definition of complete semisimplicity requires that each be completely 0 -simple or, in the last case, completely simple. (In this context, a semigroup is completely regular if, in addition, each principal factor has no zero divisors.) Such a semigroup is necessarily regular and satisfies $\mathrm{D}=\mathcal{J}$. For these and further properties, we refer the reader to [2, Chapter 6].

The congruence-free completely simple semigroups are easily seen to be the two-element right and left zero semigroups and the simple groups. Tamura [6] showed that a completely 0-simple semigroup, when represented as a Rees matrix semigroup $\mathrm{MO}(I, G, \wedge, P)$, is congruence-free if and only if $G$ is trivial and no rows, nor columns, of $P$ are identical. (Our determination of the L-CF completely 0 -simple semigroups in Sect. 4 also has this description as a consequence).

\section{Necessary conditions}

Our first result extends [1, Lemma 2.6] to semigroups in general.

Lemma 2.1 Let $\mathrm{T}$ be an equivalence relation on a semigroup $S$ and assume that $S$ is $T$-CF. If $T$ is nontrivial on the $J$-class $J$, then $T \subseteq(J \times J) \cup(J \times Q(J)) \cup(Q(J) \times J) \cup \epsilon$. In particular, $T$ is trivial on all other $\mathcal{J}-$ classes of $S$. If, further, $T \subseteq \mathcal{J}$, then $T \subseteq(J \times J) \cup \in$. 
Proof. First suppose $x, y$ are any distinct $T$-related elements of $S$, let $I(x, y)=S^{1}\{x, y\} S^{1}$, the ideal generated by $\{x, y\}$, and let $\rho$ be the Rees congruence modulo $I(x, y)$. Since $\rho \cap T \neq \epsilon, T \subseteq \rho$. Now if $a, b$ are also distinct, $T$-related elements of $S$, it follows that $a, b \in I(x, y)$. By symmetry, $x, y \in I(a, b)$, that is, $I(x, y)=I(a, b)$.

Now if $J=J x=J y$, then $I(x, y)=I(J)$ and in that case at least one of $a$ and $b$ belongs to $J$. Thus $(a, b) \in(J \times J) \cup(J \times Q(J)) \cup(Q(J) \times J)$ and the result follows.

Corollary 2.2 Let $S$ be a regular semigroup. Then:

- If $\mathrm{S}$ is $\mathrm{H}-\mathrm{CF}$, every J-class except at most one is combinatorial.

- If $\mathrm{S}$ is $\mathrm{L}-\mathrm{CF}$, every $J$-class except at most one is a right zero semigroup.

- If $S$ is J-CF, every J-class except at most one consists of a single idempotent.

Proof. The only case that requires elaboration is the second. Suppose $L$ is trivial on a $J$-class $J$ of a regular semigroup. Since every element of such a semigroup is L-related to an idempotent, $J$ consists of idempotents. Its principal factor is therefore a completely 0 -simple band, that is, the rectangular band $J$ with zero adjoined. Again since $L$ is trivial, the rectangular band is a right zero semigroup.

The extremes in this corollary are represented by triviality of the respective relations: $S$ is a combinatorial regular semigroup, a right regular band, or a semilattice, respectively.

The following series of rather technical lemmas provides the essential tools for handling all three of the cases under consideration. First recall $[2, \S 1.6]$ that the congruence generated by a pair $(a, b)$ of elements in a semigroup $S$ is described as follows: distinct elements $x, y$ are related if and only if there is a sequence of 'elementary transitions' $x=z_{0} \rightarrow z_{1} \rightarrow \cdots \rightarrow z_{n}=y$ of distinct elements of $S$; that is, for each $i,\left\{z_{i-1}, z_{i}\right\}=\left\{s_{i} a t_{i}, s_{i} b t_{i}\right\}$, for some $s_{i}, t_{i} \in S^{1}$. 
In each of the next four lemmas, $\sigma(a, b)$ denotes the congruence generated by a pair $(a, b)$ of elements of a $J$-class $J$ on the ideal $I(J)$ generated by $J$. A congruence is said to saturate a subset $A$ of a semigroup if $A$ is a union of its classes.

Lemma 2.3 Let $\mathrm{J}$ be a regular J-class of a semigroup $S$, let $a, b \in J$ and suppose that $\sigma(a, b)$ saturates $J$. If sat $\in J$ for some $s, t \in S^{1}$, then sbt $\in \mathrm{J}$. Hence if $x, y \in J$ and $(x, y)$ belongs to the congruence on $S$ generated by $(a, b)$, then the terms in any sequence of elementary transitions (as described above) also belong to $\mathrm{J}$.

Proof. If sat $\in J$, then $s a$, at $\in J$. We may write sat $=(s a)(s a)^{\prime} s a t(a t)^{\prime}(a t)$, where $(s a)^{\prime} \in V(s a),(a t)^{\prime} \in V(a t)$. Now $(s a)(s a)^{\prime} s L s a$, so $(s a)(s a)^{\prime} s \in J$; similarly $t(a t)^{\prime}(a t) \in J$. Therefore (sat, (sa)(sa)'sbt(at)'(at)) $\in \sigma(a, b)$. By saturation, $(s a)(s a)^{\prime} s b t(a t)^{\prime}(a t) \in J$, whence $s b t \in J$. The last statement then follows by induction.

Lemma 2.4 Let $\mathrm{J}$ be a J-class of a semigroup $S$ and $a, b \in J$.

(1) If $\mathrm{J}$ is a subsemigroup of $S$, then $\sigma(a, b)$ saturates $J$.

(2) If $\mathrm{J}$ is not a subsemigroup of $\mathrm{S}$ and $\sigma(a, b)$ does not saturate $J$, then the congruence generated by $(a, b)$ on the principal factor $\operatorname{PF}(J)$ is the universal relation.

Proof. In (1), if $x \in J, y \in I(J), y \neq x$ and $(x, y) \in \sigma(a, b)$, then there is a sequence $x=z_{0} \rightarrow z_{1} \rightarrow \cdots \rightarrow z_{n}=y$ of elementary transitions, as described above, with each $s_{i}, t_{i} \in I(J)$. Since $x \in\left\{s_{1} a t_{1}, s_{1} b t_{1}\right\}, s_{1}, t_{1} \in J_{1}$ and so $z_{1} \in J$. The proof then proceeds by induction.

In (2), there exist $x \in J$ and $y \in Q(J)$ such that $(x, y) \in \sigma(a, b)$.

Interpreted in $P F(J), x$ is related to 0 under the congruence generated thereon by $(a, b)$. Since $P F(J)$ is 0 -simple, every element of $J$ is related to 0 .

Lemma 2.5 Let $\mathrm{J}$ be a regular J-class of a semigroup $S$ and let $a, b \in J$. Let $\rho$ be the congruence on $J$ generated by $(a, b)$, if $J$ is a subsemigroup of $S$, or the congruence on $\operatorname{PF}(J)$ generated by $(a, b)$, otherwise. 
NOT THE PUBLISHED VERSION; this is the author's final, peer-reviewed manuscript. The published version may be accessed by following the link in the citation at the bottom of the page.

(1) If $\mathrm{J}$ is a subsemigroup of $S$, then the restriction of $\sigma(a, b)$ to $J$ coincides with $\rho$.

(2) If $\mathrm{J}$ is not a subsemigroup of $\mathrm{S}$ and $\sigma(a, b)$ saturates $\mathrm{J}$, then the restriction of $\sigma(a, b)$ to $J$ coincides with the restriction of $\rho$ to $\mathrm{J}$.

Proof. By the previous lemma, $\sigma(a, b)$ saturates $J$ in each case. Therefore Lemma 2.3 applies. In particular, it may be applied to $I(J)$ itself, so that any sequence of elementary transitions between elements of $J$ lies totally in $J$. So in each case, the first congruence is contained in the second. In the case that $J$ is a subsemigroup, the opposite inclusion is obvious; in the alternative case, the proof proceeds similarly to the argument for the original inclusion.

Lemma 2.6 Let $J$ be a nontrivial $J$-class of a semigroup $S$, with $a, b \in J$. Let $\rho$ be the congruence on $J$ generated by $(a, b)$, if $J$ is a subsemigroup of $S$, or the congruence on $\operatorname{PF}(J)$ generated by $(a, b)$, otherwise. Suppose either of the following holds:

(A) there is an idempotent $e \in J$ such that $a, b \in e S e$, or

(B) $S$ is an L-CF regular semigroup and $a \mathrm{~L} b$. If $u, v \in J$ and $(u, v)$ belongs to the congruence on $S$ generated by $(a, b)$, then $(u, v) \in \rho$.

Proof. If $J$ is not a subsemigroup of $S$ and $J$ is not saturated by $\sigma(a, b)$, then the conclusion follows from Lemma 2.4(2). Thus, in view of (1) of the same lemma, we may assume throughout that $\sigma(a, b)$ saturates $J$. Then Lemmas 2.3 and 2.5 apply. In particular, working with $\rho$ is equivalent to working with $\sigma(a, b)$ and so we do not need to distinguish between the cases according to whether or not $J$ is a subsemigroup of $S$.

With $u, v$ as stated, there is a sequence $u=z_{0} \rightarrow z_{1} \rightarrow \cdots \rightarrow z_{n}=v$, where for each $i,\left\{z_{i-1}, z_{i}\right\}=\left\{x_{i} a y_{i}, x_{i} b y_{i}\right\}, x_{i}, y_{i} \in S^{1}$. Then for each $i, z_{i} \in J, J_{x_{i}} \geq J$ and $J, J_{y_{i}} \geq J$.

(A) Here $e$ is an identity element for $a$ and $b$, so it may be assumed that $x_{i}=x_{i} e$ and $y_{i}=e y_{i}$, so that $x_{i}, y_{i} \in J$. Hence $(u, v) \in \rho$.

Semigroup Forum, Vol. 89, No. 2 (October 2014): pg. 383-393. DOI. This article is (C) Springer and permission has been granted for this version to appear in e-Publications@Marquette. Springer does not grant permission for this article to be further copied/distributed or hosted elsewhere without the express permission from Springer. 
(B) In this case, since $a \mathrm{~L} b$ it may be assumed that each $y_{i}=a^{\prime} a y_{i}$, where $a^{\prime} \in V(a)$, so each $y_{i} \in J$. Thus it suffices to show that $x_{i} a \rho x_{i} b$ for each $i$. If $x i \in J$, this is clear, so it remains to prove the following statement.

If $g$ is a (necessarily idempotent) element of $S$ such that $J_{g}>J$ and $g a, g b \in J$, then ga $\rho g b$.

Consider first the case that $g a, g b$ are again L-related.

Assuming $g a \neq g b$, from L-CF it follows that $(a, b)$ belongs to the congruence on $S$ generated by $(g a, g b)$. There is therefore a sequence $a=w_{0} \rightarrow w_{1} \rightarrow \cdots \rightarrow w_{n}=b$, where for each $i,\left\{w_{i-1}, w_{i}\right\}=\left\{s_{i}(g a) t_{i}\right.$, $\left.s_{i}(g b) t_{i}\right\}, s_{i}, t_{i} \in S^{1}$ and, for each $i, w_{i} \in J, J_{s_{i} g} \geq J$ and $t_{i} \in J$.

Now consider the sequence $g a=g w_{0} \rightarrow g w_{1} \rightarrow \cdots \rightarrow g w_{n}=g b$, where for each $i,\left\{g w_{i-1}, g w_{i}\right\}=\left\{g s_{i}(g a) t_{i}, g s_{i}(g b) t_{i}\right\}$ and, once again, each term belongs to $J$. If every $s i g \in J$, then ga $\rho g b$.

Otherwise, let $i$ be the first index such that $s_{i} g \notin J$, that is, the $\mathcal{J}$-class $J_{s_{i} g}$ is strictly above $J$. By Corollary 2.2, this $\mathcal{J}$-class is a right zero semigroup, so $s_{i} g=g s_{i} g$ (the element $s_{i} g$ is idempotent and so $g s_{i} g \mathrm{~L} s_{i} g$ ). It follows that $w_{i-1}=g w_{i-1}$. If $i=1$, then $a=g a$. If $I>1$, then since $s_{j} g \in J$ for $j<i$, the sequence $a=w_{0} \rightarrow \cdots \rightarrow w_{i-1}$ shows that $a w_{i-1}$ and the sequence $g a=g w_{0} \rightarrow \cdots \rightarrow g w_{i-1}$ shows that $g a \rho g w_{i-1}=w_{i-1}$. So a $\rho$ ga. Now if $j$ is the last index such that $s_{j} g \notin J$, then the same reasoning yields $b \rho g b$. Therefore ga $\rho g b$.

We reduce the general case to the one just considered, as follows. For convenience, put $x=g a, y=g b$ and let $x^{\prime} \in V(x), y^{\prime} \in V(y)$. Note that $y=\left(y y^{\prime} g\right) b \rho\left(y y^{\prime} g\right) a=y y^{\prime} x$. Similarly, $x x^{\prime} y \rho x$. From the former relation it follows that $y \rho y x^{\prime} x$. (As a consequence of these relations, $y y^{\prime} x, y x^{\prime} x \in J$.) We show $x \rho y x^{\prime} x$.

Let $\left(y x^{\prime} x\right)^{\prime} \in V\left(y x^{\prime} x\right)$, and let $f$ be the (idempotent) element $\left(x^{\prime} x\right)\left(y x^{\prime} x\right)^{\prime}\left(y x^{\prime} x\right)\left\llcorner y x^{\prime} x\right.$. (Since $y x^{\prime} x \in J, f \in J$.) Now (af, bf) $\in \rho$ and af $\mathrm{L} b f$ in $J$. Here $g(a f)=x f=x\left(y x^{\prime} x\right)^{\prime}\left(y x^{\prime} x\right) L y x^{\prime} x=y x^{\prime} x\left(y x^{\prime} x\right)^{\prime}\left(y x^{\prime} x\right)=y f=g(b f)$. Again, $g(a f), g(b f) \in J$. By the case previously considered, (with $a$ and $b$ replaced by af and $b f)$, these elements are $\rho$-related. That is, $y x^{\prime} x \rho$ $x\left(y x^{\prime} x\right)^{\prime}\left(y x^{\prime} x\right)$. 
It follows that $y x^{\prime} x \rho x x^{\prime} y x^{\prime} x$. But $x x^{\prime} y \rho x$, so $y x^{\prime} x \rho x$.

Proposition 2.7 Let $S$ be a regular semigroup.

If $S$ is $\mathrm{H}$-CF and the $\mathcal{J}$-class $\mathrm{J}$ of $\mathrm{S}$ is not combinatorial, then its principal factor is a [0-] simple $\mathrm{H}$-CF semigroup.

If $S$ is $L-C F$ and the $J$-class $J$ of $S$ is not a right zero semigroup, then its principal factor is a [0-] simple L-CF semigroup.

If $S$ is $J-C F$ and the $\mathcal{J}$-class $J$ of $S$ is nontrivial, then either

(i) $\mathrm{J}$ is a (simple) subsemigroup, in which case it is a congruence-free semigroup without zero;

(ii) or, otherwise, its principal factor $\operatorname{PF}(\mathrm{J})$ is a congruence-free semigroup with zero.

Proof. In each case, we consider the two situations ( $\mathrm{J}$ a subsemigroup or not) in parallel.

The first case to consider is where $S$ is $\mathrm{H}-\mathrm{CF}$. Let $T$ be a congruence on $J[P F(J)]$ that contains a pair $(a, b)$ of distinct, $\mathrm{H}$ related elements. Then, by hypothesis, any pair $(u, v)$ of $\mathrm{H}$-related elements of $J$ belongs to the congruence on $S$ generated by $(a, b)$. If $a^{\prime} \in V(b)$, then $(a, b)$ and $\left(a a^{\prime}, b a^{\prime}\right)$ generate the same congruence, with $a a^{\prime} \mathrm{H} b a^{\prime}$. Letting e denote the idempotent $a a^{\prime}$ of J, we have $a a^{\prime}$, $b a^{\prime} \in$ eSe. By Lemma 2.6(A), $(u, v)$ belongs to the congruence on $J$ [on $P F(J)]$ generated by $\left(a a^{\prime}, b a^{\prime}\right)$ and hence to $T$. Hence $\mathrm{H} \subseteq T$ and $J[P F(J)]$ is $\mathrm{H}-\mathrm{CF}$.

The second case to consider is where $S$ is L-CF. Let $T$ be a congruence on $J[P F(J)]$ that contains a pair $(a, b)$ of distinct, L-related elements. Then, by hypothesis, any pair $(u, v)$ of L-related elements of $J$ belongs to the congruence on $S$ generated by $(a, b)$. By Lemma 2.6(B), $(u, v)$ belongs to the congruence on $J$ [on $P F(J)$ ] generated by $(a, b)$ and hence to $T$. Hence $L \subseteq T$ and $J[P F(J)]$ is $L-C F$.

The final case to consider is where $S$ is $\mathcal{J}$-CF (and thereby L$\mathrm{CF}$ ). Let $T$ be a nontrivial congruence on $J[P F(J)]$. Then, again by hypothesis, any pair $(u, v)$ of elements of $J$ belongs to the congruence 
on $S$ generated by $(a, b)$. Consider first the possibility that $e t$ a for some $e \in E J$ and $a \in e J e, e \neq a$. By Lemma 2.6(A), $(u, v)$ belongs to the congruence on $J$ [on $\operatorname{PF}(J)]$ generated by $(e, a)$ and hence to $T$.

From now, it may be assumed that for each $e \in E_{J}$, eT $\cap$ eJe $=$ $\{e\}$. It follows that $e T$ is a rectangular band. (While this fact is surely well known, we will prove it after completing the proof of the theorem.) Let $a, b$ be distinct $T$-related members of $J$, with respective inverses $a^{\prime}, b^{\prime}$. Then $a a^{\prime} T b a^{\prime}$ and $b^{\prime} b T b^{\prime} a$. If equality holds in both cases, then $a=b a^{\prime} a$, and so $a=b b^{\prime} a=b$, a contradiction. In view of the assumption made above, there exist distinct $T$-related idempotents $e, f$ in $J$. By applying duality, if necessary, it may be assumed that $e\llcorner f$.

By Lemma 2.6(B), $(u, v)$ belongs to the congruence on $J$ [on $P F(J)]$ generated by $(a, b)$ and hence to $T$. Hence if $J$ is a subsemigroup, then it is congruence-free; and if not, then $\operatorname{PF}(J)$ is congruence-free.

The following was used in the proof of the theorem.

Lemma 2.8 For a congruence $\rho$ on a regular semigroup $S$, the following are equivalent:

(a) $e \rho$ is a rectangular band, for each idempotent $e$;

(b) $e \rho \cap e S e=\{e\}$ for each idempotent $e$.

Proof. The implication (a) $\Rightarrow(b)$ is obvious. To prove the converse, suppose e $\rho$ a. Then $e \rho$ eae $\in e S e$, so $e=e a e$; similarly, $e=e a^{2} e$; further, if $a^{\prime}$ is any inverse of $a$, then since e $\rho$ eaa'ae $\rho$ ead'e, $e=e a a^{\prime} e$ and so $a a^{\prime} e \in E_{S}$.

From e $\rho$ a it also follows that ( $\left.a a^{\prime}\right) e a^{\prime} \rho a a^{\prime}$ and so $a a^{\prime}=a a^{\prime} e a^{\prime}$, whence $a=a a^{\prime} e a^{\prime} a$ and $a e=\left(a a^{\prime} e\right)^{2}=a a^{\prime} e$. Dually, ea $=e a^{\prime} a$ and so $a=$ aea. Finally, $a^{2}=a e a^{2} e a=a e a=a$.

We have shown that $e \rho \subseteq E_{S}$; and also that if e $\rho f \in E_{S}$, then $e=$ efe. Since, similarly, $f=f$ e $f$, e $\rho$ is a rectangular band.

Semigroup Forum, Vol. 89, No. 2 (October 2014): pg. 383-393. DOI. This article is (C Springer and permission has been granted for this version to appear in e-Publications@Marquette. Springer does not grant permission for this article to be further copied/distributed or hosted elsewhere without the express permission from Springer. 
NOT THE PUBLISHED VERSION; this is the author's final, peer-reviewed manuscript. The published version may be accessed by following the link in the citation at the bottom of the page.

\section{Sufficiency}

Theorem 3.1 Let $S$ be a regular semigroup.

(1) $S$ is L-CF if and only if either it is a right regular band, or every $\mathrm{J}$-class but one is a right zero semigroup and the principal factor of the remaining $\mathrm{J}$-class $\mathrm{J}$ is a [0-] simple L-CF semigroup.

(2) $\mathrm{S}$ is $\mathrm{J}$-CF if and only if either it is a semilattice, or every J-class but one consists of a single idempotent and, for the remaining J-class $J$, either

(i) $\mathrm{J}$ is a (simple) subsemigroup, in which case it is a congruence-free semigroup without zero;

(ii) or, otherwise, its principal factor $P F(J)$ is a congruence-free semigroup with zero.

Proof. In each case, only sufficiency remains to be established, by virtue of Corollary 2.2 and Proposition 2.7.

(1) If $S$ is a right regular band, then $L$ is trivial and so $S$ is clearly L-CF. Otherwise, let $J$ be as stated, so that the only nontrivial $L$-classes of $S$ are contained within it. Let $\rho$ be a congruence on $S$ that meets $L$ nontrivially. By intersecting $\rho$ with the Rees congruence modulo $I(J)$, if necessary, it may be assumed that $\rho$ is contained in that Rees congruence. It suffices to show that the L-relation in $J$ is contained within $\rho$.

Consider first the case that $\rho$ saturates $J$. If $J$ is a subsemigroup, $\rho$ induces a congruence on it, by restriction. Otherwise, $\rho$ induces a congruence on the principal factor $P F(J)$ : the union of the restriction of $\rho$ to $J$ with the pair $(0,0)$. In each case, the induced congruence meets $L$ nontrivially and thus, by assumption, contains $L$. Since all remaining $L$-classes of $S$ are trivial, $\rho$ itself contains $L$.

Alternatively, there exist $x \in J, y \in Q(J)$ such that $x \rho y$. We must show that if $a \mathrm{~L} b$ in $J$, then $a \rho b$. Let $a^{\prime} \in V(a)$. Then since $L$ is $a$ right congruence, $a a^{\prime} \mathrm{L} b a^{\prime}$, where $a a^{\prime} \in E J$. Also $a=\left(a a^{\prime}\right) a, b=$ $\left(b a^{\prime}\right) a$. Thus it suffices to show that if $e \mathrm{~L} b$ in $J$, where $e \in E J$, then e $\rho$ $b$. granted for this version to appear in e-Publications@Marquette. Springer does not grant permission for this article to be further copied/distributed or hosted elsewhere without the express permission from Springer. 
Recall that each $\mathcal{J}$-class in $Q(J)$ is a right zero semigroup. Thus $Q(J)$ is a right regular band. Now $e=s x t$ for some $s, t \in S^{1}$, so e $\rho$ syt $\in Q(J)$. Put $f=s y t$. Now e $\rho f \rho$ efe $\in Q(J)$, so without loss of generality $f<e$. Further, since $\mathrm{L} b, b=b e \rho b f$, where $b f \mathrm{~L}$ ef $=f$. Since $\mathrm{L}$ is trivial on $Q(J), b f=f$ and therefore $e, b$, as required.

Observe that only the following assumptions were used in the last two paragraphs: that there exist $x \in J, y \in Q(J)$ such that $x \rho y$ and that $Q(J)$ be a right regular band.

(2) The proof parallels closely that for L. Again, if $S$ is a semilattice, $\mathcal{J}$ is trivial and $S$ is clearly $\mathcal{J}-C F$. Otherwise, let $J$ be as stated. Let $\rho$ be a congruence on $S$ that meets $\mathcal{J}$ nontrivially. Again, it may be assumed that $\rho$ is contained in the Rees congruence modulo $I(J)$. It is required to show that all members of $J$ are related under $\rho$.

Again, consider first the case that $\rho$ saturates $J$. Then it induces a nontrivial congruence on either $J$, in case (i), or on $\operatorname{PF}(J)$, in case (ii). By hypothesis, the induced congruence identifies all members of $J$ and so the same is true for $\rho$.

In the alternative case, once again there exist $x \in J, y \in Q(J)$ such that $x \rho y$. Further, since $Q(J)$ is a semilattice, it is a right regular band. Thus the proof of the corresponding case in (1) shows that Lrelated elements in $J$ are contained in $\rho$. Since all other $\mathcal{J}$-classes are trivial, $\rho$ contains L. By duality, it also contains $\mathrm{R}$ and thus contains $\mathrm{D}$. Therefore it contains the congruence generated by $D$. But on any regular semigroup, the congruences generated by $D$ and $\mathcal{J}$ coincide (each is the least semilattice congruence: this may be easily proven, or see [4]), so $\rho$ contains $\mathcal{J}$, as required.

Since every congruence-free semigroup is ([0-] simple and) also $\mathrm{L}-\mathrm{CF}$, it is difficult to imagine a more concrete description of the latter property for [0-] simple regular semigroups in general. However, it would be of interest to find a 0 -simple L-CF regular semigroup that is not congruence-free and not completely 0 -simple. (It will be seen in the next section that such examples exist in the completely 0 -simple situation).

Semigroup Forum, Vol. 89, No. 2 (October 2014): pg. 383-393. DOI. This article is @ Springer and permission has been granted for this version to appear in e-Publications@Marquette. Springer does not grant permission for this article to be further copied/distributed or hosted elsewhere without the express permission from Springer. 
We do not know if the analogue of Theorem 3.1 also holds for $\mathrm{H}-\mathrm{CF}$. As remarked in the Introduction, we have not considered the property D-CF at all. Several interesting questions arise in that regard. For instance, does the analogue of the above theorem hold? Is D-CF equivalent to $\mathcal{J}$-CF for regular semigroups?

\section{Complete semisimplicity}

Congruence-free completely [0-] simple semigroups are well known and so Theorem 3.1 can be made much more precise for completely semisimple semigroups. Further, under this hypothesis, necessary and sufficient conditions for $\mathrm{H}-\mathrm{CF}$ are also found. In fact, the analogue of Theorem 3.1 also holds for $\mathrm{H}-\mathrm{CF}$ in this restricted situation. In this section we will state a separate theorem for each property.

When specialized to completely regular semigroups, the three theorems reduce precisely to [1, Theorems 3.3 and 4.5, Corollary 4.7], respectively, by omitting reference to principal factors that possess zero divisors.

Theorem 4.1 Let S be a completely semisimple semigroup. Then S is $\mathrm{H}-\mathrm{CF}$ if and only if either:

(a) $S$ is combinatorial, or

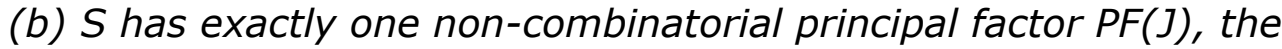
maximal subgroups of which are simple groups; equivalently, the principal factor is $\mathrm{H}-\mathrm{CF}$.

Proof. First the proof of necessity must be completed. Assuming that $S$ is not combinatorial, then by Corollary $2.1, \mathrm{H}$ is nontrivial on a single $\mathcal{J}$-class $J$. Assume $P F(J)$ is completely 0 -simple (the completely simple case being similar, but easier), with nonzero maximal subgroups isomorphic to $G$, say.

The congruences on a completely [0-] simple semigroup are well described (refer, for instance, to [3]). All that is needed for this paper is that the congruences contained in $\mathrm{H}$ - the idempotentseparating ones-are in one-one correspondence with the normal 
subgroups of the structure group $G$ (that is, the isomorphism class of the nonzero maximal subgroups).

In particular, $\mathrm{H}$ itself is a congruence. So $\mathrm{H}-\mathrm{CF}$ is equivalent to the property that $\mathrm{H}$ properly contains no nontrivial congruences. In conjunction with the previous paragraph, it follows that such a semigroup is $\mathrm{H}-\mathrm{CF}$ if and only if its nonzero maximal subgroups are simple. In conjunction with Proposition 2.7, that completes the proof of necessity.

To prove the converse, we must first review the congruences on $P F(J)=I(J) / Q(J)=I(J) / \rho_{Q(J)}$, where $\rho_{Q(J)}$ denotes the Rees congruence modulo $Q(J)$. The non-universal congruences are in one-one correspondence with those congruences on $I(J)$ that saturate $J$ and identify all elements of $Q(J)$ (in effect, by taking the union of the restriction of each congruence to $J$ and then the union of that equivalence relation with $\left.\rho_{Q(J)}\right)$. In particular, in our situation, $\mathrm{H}$ on $\operatorname{PF}(J)$ corresponds to the congruence $\mathrm{H} \cup \rho_{Q(J)}$ on $I(J)$.

Now let $\rho$ be a congruence on $S$ that meets $H$ nontrivially, within the $\mathcal{J}$-class $J$. The intersection of $\rho$ with $\mathrm{H} \cup \rho_{Q(J)}$ saturates $J$ and so induces a congruence on $\operatorname{PF}(J)$, whose classes are just the classes of $\rho \cap \mathrm{H}$ on $J$, together with the zero element (if one exists). By assumption, this induced congruence must be all of $\mathrm{H}$. Thus, in $S$, $\mathrm{H} \subseteq \rho$.

We next consider $\mathcal{J}-\mathrm{CF}$, since the congruence-free completely [0-] simple semigroups are already well known (see the Introduction, but this description also follows immediately from that for L-CF proven below, in conjunction with its dual) and so the description is direct from Theorem 3.1.

Theorem 4.2 Let $S$ be a completely semisimple semigroup. Then $S$ is $\mathcal{J}-C F$ if and only if one of the following holds:

(a) $S$ is a semilattice;

(b) every $\mathcal{J}$-class but one of $S$ consists of a single idempotent, and the remaining $\mathcal{J}$-class $\mathrm{J}$ either:

Semigroup Forum, Vol. 89, No. 2 (October 2014): pg. 383-393. DOI. This article is @ Springer and permission has been granted for this version to appear in e-Publications@Marquette. Springer does not grant permission for this article to be further copied/distributed or hosted elsewhere without the express permission from Springer. 
NOT THE PUBLISHED VERSION; this is the author's final, peer-reviewed manuscript. The published version may be accessed by following the link in the citation at the bottom of the page.

(i) is a nontrivial simple group;

(ii) is a rectangular band with at most two elements;

(iii) or has a combinatorial principal factor $\operatorname{PF}(\mathrm{J})$, with zero divisors, the Rees matrix representation of which contains no identical columns and no identical rows.

Recall [2] that a right group is a completely simple semigroup with exactly one R-class, equivalently a semigroup isomorphic to the direct product of a right zero semigroup with a group.

Theorem 4.3 Let $S$ be a completely semisimple semigroup. Then $S$ is L-CF if and only if one of the following holds:

(a) $S$ is a right regular band;

(b) every $\mathcal{J}$-class but one of $S$ is a right zero semigroup and the remaining J -class $\mathrm{J}$ either:

(i) is a right group, whose maximal subgroups are nontrivial simple groups;

(ii) is a rectangular band with at most two $R$-classes;

(iii) or has a combinatorial principal factor $\operatorname{PF}(\mathrm{J})$, with zero divisors, the Rees matrix representation of which contains no identical columns.

Proof. To complete the proof, it needs to be shown that (a) the completely simple L-CF semigroups $S$ are those in (i) and (ii), and (b) the completely 0 -simple L-CF semigroups $S$ are those in (iii) (cf the corresponding case in the previous theorem).

The first case is covered by [1], but we outline a proof for completeness' sake. First, since L-CF implies H-CF, the maximal subgroups are simple, by Theorem 4.1. Second, if the maximal subgroups are nontrivial, $R$ meets $L$ nontrivially, so necessarily $L \subseteq R$, that is, $\mathrm{L}=\mathrm{H}$ and in that case the semigroup is a right group. If the maximal subgroups are trivial, $S$ is a rectangular band. That there are

Semigroup Forum, Vol. 89, No. 2 (October 2014): pg. 383-393. DOI. This article is @ Springer and permission has been granted for this version to appear in e-Publications@Marquette. Springer does not grant permission for this article to be further copied/distributed or hosted elsewhere without the express permission from Springer. 
at most two R-classes follows from the argument in the next case, below. The converse is also easily disposed of.

The second case is essentially a one-sided version of the characterization of the congruence-free completely 0 -simple semigroups and could be deduced from any of the familiar descriptions of the congruences on completely 0 -simple semigroups. However, we provide an elementary argument.

Suppose that $T$ is a combinatorial, completely 0 -simple L-CF semigroup with zero divisors, whose Rees matrix representation includes two identical columns. Translated into the language of Green's relations, this means that the associated $\mathrm{R}$-classes have the property that when intersected with any nonzero L-class, either each $\mathrm{H}$-class consists of an idempotent or each consists of a nonidempotent. Choose L-related idempotents $e, f$ from these two R-classes and let $\rho$ be the congruence generated by the pair $(e, f)$. Let $x=$ set $\rightarrow s f t=y$ be an elementary transition (see the discussion preceding Lemma 2.3), where $x \neq 0$. If $s \neq 1$, then set $\mathrm{R} s \mathrm{R}$ sft and set $\mathrm{L}$ et $\mathrm{L} f \mathrm{ft}$ sft, that is, set $=$ sft. As a result, if $x \rho \neq\{x\}, x=$ et or $x=f t$, so that $x \in R_{e} \cup R_{f}$.

Now by hypothesis, $L \subset \rho$, so if $x \in L_{e}, x=e$ or $x=f$. Thus $T=$ $R_{e} \cup R_{f} \cup\{0\}$. But each L-class of $T$ contains some idempotent and the assumption on $R_{e}$ and $R_{f}$ ensures that, in fact, each L-class consists of two idempotents. In other words, $T$ is a rectangular band, comprising two R-classes, with adjoined zero. But this contradicts the assumption that $T$ has zero divisors.

To prove the converse, let $\rho$ be a congruence on $T$ that meets $\mathrm{L}$ nontrivially, say $(x, y) \in \rho \cap \mathrm{L}$. Since $J$ is combinatorial, $R_{x} \neq R_{y}$. Interpreting the hypothesis in terms of the previous paragraph, there exists an idempotent $g \in R_{x}$ such that the H-class $R_{y} \cap L_{g}$ does not contain an idempotent. Then $x=g x \rho g y=0$. By 0 -simplicity, $\rho$ must in fact by the universal relation. Therefore $T$ is $L-C F$.

\section{References}

1. Yu, H., Wang, Z., Wu, T., Ye, M.: Classification of some $T$-congruence-free completely regular semigroups, Semigroup Forum 84 (2012), 308322.

Semigroup Forum, Vol. 89, No. 2 (October 2014): pg. 383-393. DOI. This article is (C) Springer and permission has been granted for this version to appear in e-Publications@Marquette. Springer does not grant permission for this article to be further copied/distributed or hosted elsewhere without the express permission from Springer. 
NOT THE PUBLISHED VERSION; this is the author's final, peer-reviewed manuscript. The published version may be accessed by following the link in the citation at the bottom of the page.

2. Clifford, A.H., Preston, G.B.: The algebraic theory of semigroups, Amer. Math. Soc., Providence, RI I(1961), II(1967).

3. Howie, J.M.: Fundamentals of semigroup theory. Clarendon Press, Oxford 1995.

4. Howie, J.M., Lallement, G.: Certain fundamental congruences on a regular semigroup. Proc. Glasgow Math. Assoc. 7 (1966), 145-156.

5. Sutov, E.G.: Embeddings of semigroups into simple and complete semigroups. Mat. Sb. (NS) 62 (104) (1963), 496-511.

6. Tamura, T.: Indecomposable completely simple semigroups except groups, Osaka Math. J. 8 (1956), 35-42.

\section{About the Authors}

Peter R. Jones : Department of Mathematics, Statistics and Computer Science, Marquette University, Milwaukee, WI 53201, USA.

email: peter.jones@marquette.edu 